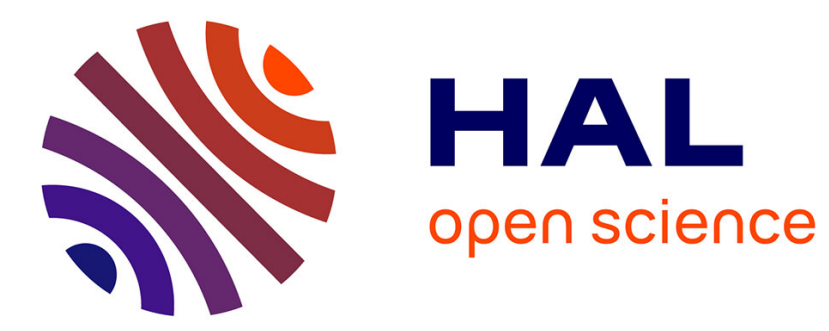

\title{
Magnetic susceptibility measurements as a probe of spin transfer driven magnetization dynamics
}

Weiwei Lin, J Cucchiara, C Berthelot, Thomas Hauet, Y Henry, J. A. Katine, Eric E. Fullerton, S Mangin

\section{- To cite this version:}

Weiwei Lin, J Cucchiara, C Berthelot, Thomas Hauet, Y Henry, et al.. Magnetic susceptibility measurements as a probe of spin transfer driven magnetization dynamics. Applied Physics Letters, 2010, 10.1063/1.3454782 . hal-01345347

\section{HAL Id: hal-01345347 \\ https://hal.science/hal-01345347}

Submitted on 13 Jul 2016

HAL is a multi-disciplinary open access archive for the deposit and dissemination of scientific research documents, whether they are published or not. The documents may come from teaching and research institutions in France or abroad, or from public or private research centers.
L'archive ouverte pluridisciplinaire HAL, est destinée au dépôt et à la diffusion de documents scientifiques de niveau recherche, publiés ou non, émanant des établissements d'enseignement et de recherche français ou étrangers, des laboratoires publics ou privés. 


\title{
Magnetic susceptibility measurements as a probe of spin transfer driven magnetization dynamics
}

\author{
Weiwei Lin (林维维), ${ }^{1}$ J. Cucchiara, ${ }^{1}$ C. Berthelot, ${ }^{1}$ T. Hauet, ${ }^{1}$ Y. Henry, ${ }^{2}$ J. A. Katine, ${ }^{3}$ \\ Eric E. Fullerton, ${ }^{4}$ and S. Mangin ${ }^{1, a}$ ) \\ ${ }^{1}$ Institut Jean Lamour, Nancy-Université, Vandoeuvre-lès-Nancy 54506, France \\ ${ }^{2}$ Institut de Physique et Chimie des Matériaux de Strasbourg, Université de Strasbourg, \\ Strasbourg 67037, France \\ ${ }^{3}$ San Jose Research Center, Hitachi Global Storage Technologies, San Jose, California 95135, USA \\ ${ }^{4}$ Department of Electrical and Computer Engineering, Center for Magnetic Recording Research, \\ University of California, San Diego, La Jolla, California 92093, USA
}

(Received 28 January 2010; accepted 16 May 2010; published online 23 June 2010)

\begin{abstract}
An experimental technique has been developed to characterize spin-transfer driven magnetization dynamics. It was tested on a nanopillar spin valve with perpendicular anisotropy by measuring the nanopillar voltage under ac injected current $(\mathrm{d} V / \mathrm{d} I)$, and ac magnetic field $(\mathrm{d} V / \mathrm{d} H)$. Both the amplitude and the sign of the signals are different which reveals the different influences of the current and the field on the magnetization dynamics. Comparison between experiments and macrospin simulation shows that $\mathrm{d} V / \mathrm{d} H$ measurements reveal the presence of a "canted state" demonstrating that $\mathrm{d} V / \mathrm{d} H$ and $\mathrm{d} V / \mathrm{d} I$ measurements are complementary techniques to probe magnetic states and their dynamics. (C) 2010 American Institute of Physics. [doi:10.1063/1.3454782]
\end{abstract}

It is now well established that magnetization switching or precession can be induced by spin polarized injected current due to the spin transfer torque (STT) effect. ${ }^{1,2}$ This topic has been extensively studied because of the interesting physics and the potential applications for spin transfer magnetic random access memories and high-frequency oscillators. ${ }^{3-10}$ Most of devices studied are nanopillar spin valves or tunnel junctions consisting of a hard magnetic layer as a polarizer for incoming electrons and a soft one as a free layer. ${ }^{3-10}$ The spin angular momentum is transferred by an injected current (dc) generating a torque on the free layer magnetization. For a small or moderate injected current, hysteretic magnetization switching can take place. For certain combination of current and magnetic field, the STT may balance the damping torque and give rise to steady magnetization precession in the gigahertz frequency regime. ${ }^{1,6}$ Furthermore, other magnetic states and dynamics have also been identified such as fluctuations, ${ }^{3}$ canted states, ${ }^{7}$ and domains states. ${ }^{8}$

The most common experiments for characterizing the dynamical magnetic states consist in recording the differential resistance $\mathrm{d} V / \mathrm{d} I$, i.e., detecting the ac voltage response to a small alternating current with a frequency (e.g., $1 \mathrm{kHz}$ ) much lower than the magnetization precession. ${ }^{3-10}$ The differential resistance can be written as

$$
\mathrm{d} V / \mathrm{d} I=R+I \mathrm{~d} R / \mathrm{d} I
$$

The first term is the dc resistance which directly probes the magnetoresistance (MR) effect. The second term probes the reversible processes such as steady magnetization precession, leading to reversible changes in resistance as a function of current.

Besides the polarized current, magnetization dynamics are also influenced by the magnetic field and temperature. For instance in the case of steady state precession, the magnetization angle can be modified by an ac magnetic field

${ }^{a)}$ Electronic mail: stephane.mangin@ijl.nancy-universite.fr. or alternative temperature, ${ }^{11-13}$ which are still under discussion. ${ }^{14}$ Here we addressed the voltage response to an ac magnetic field perturbation. To probe the magnetic acsusceptibility, we measured $\mathrm{d} V / \mathrm{d} H$ given by

$$
\mathrm{d} V / \mathrm{d} H=I \mathrm{~d} R / \mathrm{d} H+R \mathrm{~d} I / \mathrm{d} H .
$$

The first term is related to the reversible magnetic processes induced by the magnetic field and the second term results from the ac current induced by the ac magnetic field. It should be noted that in the case of low frequency excitation, the voltage response to reversible magnetic process is inphase with the ac magnetic field excitation. However, the current variation in response to the ac-magnetic field is an inductive phenomena leading to an out-of-phase response, i.e., a $90^{\circ}$ phase shift. This phase property allowed us to distinguish the two terms.

In this letter, we report a comparative study on the spintransfer-driven magnetization reversal and dynamics in a nanopillar spin valve with perpendicular magnetic anisotropy (PMA) using $R, \mathrm{~d} V / \mathrm{d} I$, and $\mathrm{d} V / \mathrm{d} H$ measurements. Both voltage responses to an ac magnetic field parallel $\left(\mathrm{d} V / \mathrm{d} H_{\|}\right)$ and perpendicular $\left(\mathrm{d} V / \mathrm{d} H_{\perp}\right)$ to the dc magnetic field were measured. Dips or peaks in $\mathrm{d} V / \mathrm{d} I, \mathrm{~d} V / \mathrm{d} H_{\|}$, and $\mathrm{d} V / \mathrm{d} H_{\perp}$ exhibit distinct features where some magnetic states are revealed by $\mathrm{d} V / \mathrm{d} H_{\|}$and $\mathrm{d} V / \mathrm{d} H_{\perp}$ while being almost absent in the $\mathrm{d} V / \mathrm{d} I$ signal.

The spin valve studied here, as reported earlier, ${ }^{7}$ consists in a $\operatorname{Pt}(30 \AA) /[\operatorname{Co}(2.5 \AA) / \operatorname{Pt}(5.2 \AA)]_{4} / \operatorname{Co}(2.5 \AA) /$ $[\mathrm{Ni}(6 \mathrm{~A}) / \mathrm{Co}(1 \AA)]_{2}$ reference layer and a $[\mathrm{Co}(1 \AA) /$ $\mathrm{Ni}(6 \mathrm{~A})]_{2} / \mathrm{Co}(2 \AA) / \operatorname{Pt}(30 \AA)$ free layer spaced by a $40 \AA$ $\mathrm{Cu}$ layer. $\mathrm{A} \mathrm{Ta}(50 \AA) / \mathrm{Cu}(300 \AA)$ film acts as the bottom lead and a $\mathrm{Cu}(200 \AA) / \mathrm{Ta}(50 \AA)$ film connects the top of the pillar to an $\mathrm{Au}$ top lead. Both the reference and the free layer are PMA. The multilayer was then patterned to a 50 $\times 200 \mathrm{~nm}^{2}$ ellipse.

The transport measurements were performed at room temperature. A dc magnetic field $H_{\mathrm{dc}}$ up to $1.5 \mathrm{~T}$ was applied 

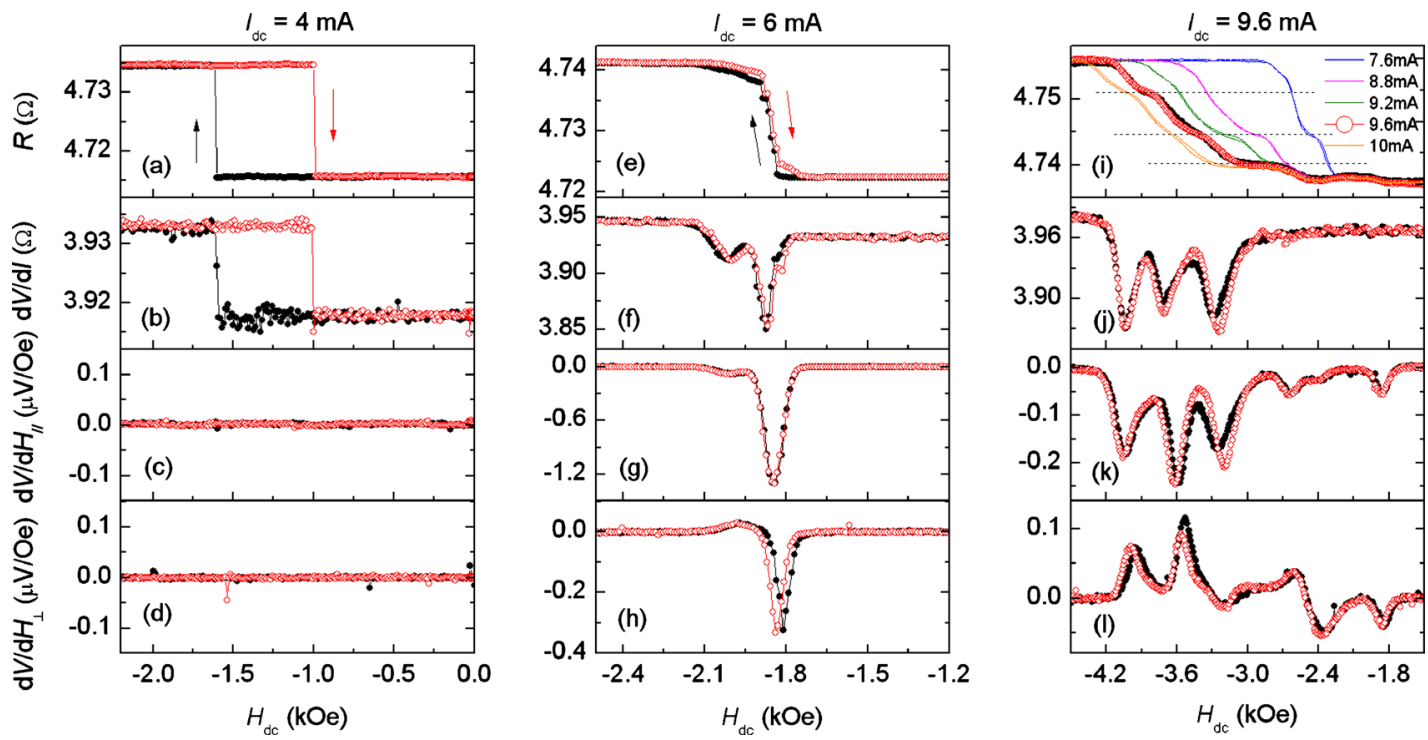

FIG. 1. (Color online) A series of the typical curves of $R, \mathrm{~d} V / \mathrm{d} I, \mathrm{~d} V / \mathrm{d} H_{\|}$, and $\mathrm{d} V / \mathrm{d} H_{\perp}$ as a function of $H_{\mathrm{dc}}$ for various $I_{\mathrm{dc}}$ [ [(a)-(d) $] I_{\mathrm{dc}}=4 \mathrm{~mA}$. [(e)-(h)] $I_{\mathrm{dc}}=6 \mathrm{~mA}$. [(i) $\left.-(1)\right] I_{\mathrm{dc}}=9.6 \mathrm{~mA}$. The curves with black solid circles refer to the magnetic field descending branches and those with red open circles stand for the magnetic field ascending branches. The $R$ as a function of $H_{\mathrm{dc}}$ for various $I_{\mathrm{dc}}$ between 7.2 and $10 \mathrm{~mA}$ are also shown in (i).

perpendicular to the film plane. The dc current $I_{\mathrm{dc}}$ was injected from a current source and the dc voltage $V_{\mathrm{dc}}$ was measured with a nanovoltmeter, allowing us to determine the resistance $R=V_{\mathrm{dc}} / I_{\mathrm{dc}}$. The positive current is defined as the one flowing from the free layer to the reference layer. To measure $\mathrm{d} V / \mathrm{d} I$, a $50 \mu \mathrm{A}$ current at a frequency $f=1 \mathrm{kHz}$ was applied and the generated voltage at the same frequency was measured with a lock-in amplifier. To probe $\mathrm{d} V / \mathrm{d} H_{\|}$and $\mathrm{d} V / \mathrm{d} H_{\perp}$, a 40 Oe magnetic field at $f=10 \mathrm{~Hz}$ was generated by a pair of Helmholtz coils either parallel $(\mathrm{P})$ or perpendicular to the dc magnetic field, respectively. The resulting ac voltage responses at the same frequency were probed by the lock-in amplifier and only the in-phase voltage responses were considered to define $\mathrm{d} V / \mathrm{d} H_{\|}$and $\mathrm{d} V / \mathrm{d} H_{\perp}$. Most of the measurements focus on large negative field and large positive current region where free layer precession is expected. ${ }^{6}$

Figure 1 shows a series of the typical curves of $R$, $\mathrm{d} V / \mathrm{d} I, \mathrm{~d} V / \mathrm{d} H_{\|}$, and $\mathrm{d} V / \mathrm{d} H_{\perp}$ as a function of $H_{\mathrm{dc}}$ for various $I_{\mathrm{dc}}$. Initially, the spin valve was saturated in a positive magnetic field to set the magnetization of the free layer $\mathrm{P}$ to that of the reference layer resulting in a low resistance $R_{\mathrm{P}}$. The dc magnetic field was swept down to $-5 \mathrm{kOe}$, where the magnetization of the free layer is antiparallel (AP) to that of the reference layer, leading to a high resistance $R_{\mathrm{AP}}$, and then swept back to $1 \mathrm{kOe}$, where the configuration is back to $\mathrm{P}$. For $I_{\mathrm{dc}}=4 \mathrm{~mA}$ (corresponding the current density $J \sim 4$ $\times 10^{7} \mathrm{~A} / \mathrm{cm}^{2}$ ), shown in Figs. 1(a)-1(d), along the magnetic field descending branch, $R$ jumps sharply from $R_{\mathrm{P}}$ to $R_{\mathrm{AP}}$ at $H_{\mathrm{dc}}=-1.6 \mathrm{kOe}$, and falls to $R_{\mathrm{P}}$ at $H_{\mathrm{dc}}=-0.99 \mathrm{kOe}$ for the ascending branch. This measurement shows the hysteretic switching of the free layer magnetization between $\mathrm{P}$ and AP configuration. The $\mathrm{d} V / \mathrm{d} I$ versus $H_{\mathrm{dc}}$ curves display similar hysteresis loops due to the absence of reversible magnetic process induced by the current, i.e., here only the $R$ term remains in Eq. (1). The $H_{\mathrm{dc}}$ dependences of both $\mathrm{d} V / \mathrm{d} H_{\|}$and $\mathrm{d} V / \mathrm{d} H_{\perp}$ show only a flat baseline meaning that almost no reversible magnetic process is induced by the magnetic field.

For $I_{\mathrm{dc}}=6 \mathrm{~mA}$, shown in Figs. $1(\mathrm{e})-1(\mathrm{~h})$, no obvious hysteresis is observed. $R$ changes rapidly for the $H_{\mathrm{dc}}$ between -1.80 and -1.87 kOe but slowly between -1.87 and $-2.10 \mathrm{kOe} . \mathrm{d} V / \mathrm{d} I$ measurements show a large dip at $H_{\mathrm{dc}}=-1.87 \mathrm{kOe}$ and a small one at $H_{\mathrm{dc}}=-2.01 \mathrm{kOe}$ related to reversible magnetic processes. The $\mathrm{d} V / \mathrm{d} H_{\|}$signal shows negative peaks similar to $\mathrm{d} V / \mathrm{d} I$. The $\mathrm{d} V / \mathrm{d} H_{\perp}$ however shows both negative and positive peaks.

For $I_{\mathrm{dc}}=9.6 \mathrm{~mA}$, shown in Figs. 1(i) $-1(1)$, there are more dips or peaks in $\mathrm{d} V / \mathrm{d} I, \mathrm{~d} V / \mathrm{d} H_{\|}$, and $\mathrm{d} V / \mathrm{d} H_{\perp}$ in a wide $H_{\mathrm{dc}}$ range, corresponding to the different jumps in $R .^{7}$ The amplitude of these peaks are different for $\mathrm{d} V / \mathrm{d} I, \mathrm{~d} V / \mathrm{d} H_{I}$ and $\mathrm{d} V / \mathrm{d} H_{\perp}$ showing the different influence of current and magnetic field on the magnetization dynamics. It is interesting that $\mathrm{d} V / \mathrm{d} H_{\|}$and $\mathrm{d} V / \mathrm{d} H_{\perp}$ exhibit some small peaks for the $H_{\mathrm{dc}}$ between -1.66 and $-2.83 \mathrm{kOe}$ corresponding to the undulation in $R$, while they are not visible in $\mathrm{d} V / \mathrm{d} I$.

The $R$ as a function of $H_{\mathrm{dc}}$ for various $I_{\mathrm{dc}}$ between 7.2 and $10 \mathrm{~mA}$ are also plotted in Fig. 1(i). It can be seen that the curves are reversible but also show plateaus that are separated by similar giant magnetoresistance (GMR) steps. Assuming these intermediate resistance plateaus represent precession states, these results would tend to show that magnetization prefers to precess with particular angles. Indeed the spin valve GMR value depends on the relative angle between the free layer moments and the reference layer that is out of the film plane. In this case the precession angle $\varphi$ can be roughly calculated from $\cos \varphi=1-2[R(\varphi)$ $\left.-R_{\mathrm{P}}\right] /\left(R_{\mathrm{AP}}-R_{\mathrm{P}}\right) .{ }^{15}$ The reasons of those particular angles are not known and could be due to particular aspect of the nanopillar shape as the details of these steps varies from sample to sample. An alternate interpretation could be in terms of incoherent precession (spin waves).

Nevertheless those steps and plateaus give rise to dips or peaks in $\mathrm{d} V / \mathrm{d} I$ and $\mathrm{d} V / \mathrm{d} H$ measurements. It should be noted that different features in the dips or peaks for $\mathrm{d} V / \mathrm{d} I$, $\mathrm{d} V / \mathrm{d} H_{\|}$, and $\mathrm{d} V / \mathrm{d} H_{\perp}$ indicate the influences of current and magnetic field on the magnetization precession. The magnetization dynamics results from the competition between the torque due to the magnetic field, the damping and the spin torque. In a macrospin approach magnetization precession appears when the spin torque balances the damping, a 

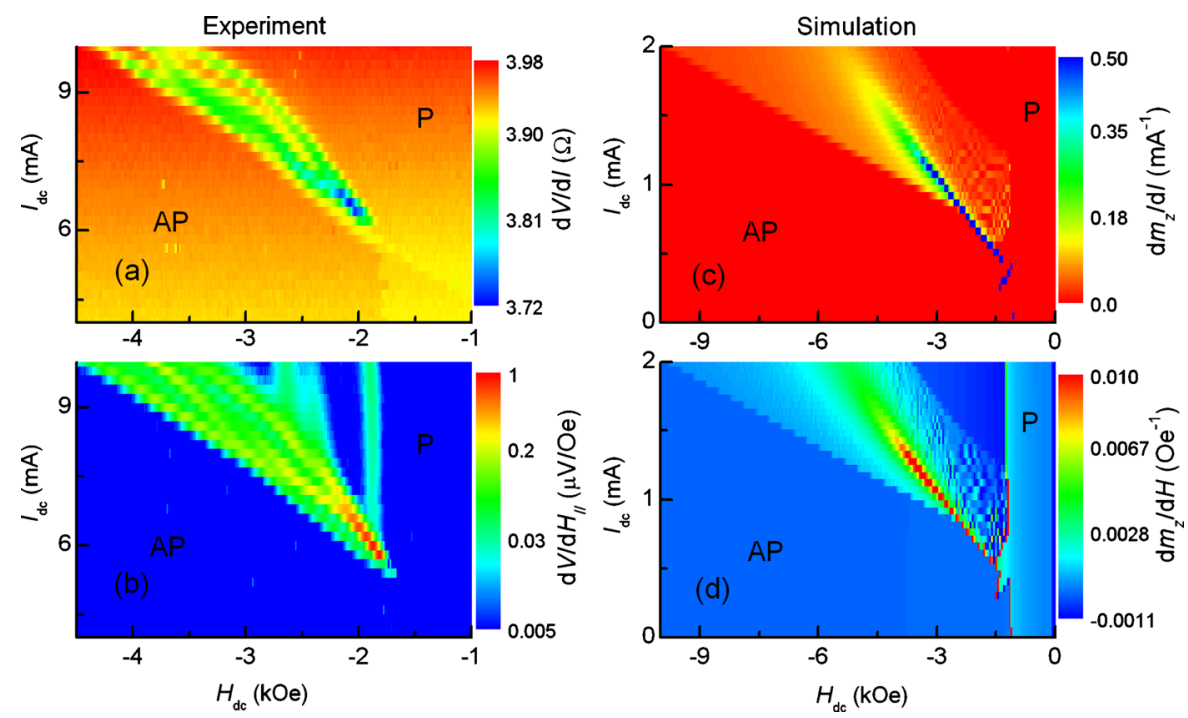

FIG. 2. (Color online) $[(a)$ and (b) The $\left(H_{\mathrm{dc}}, I_{\mathrm{dc}}\right)$ phase diagrams for the average values of $\mathrm{d} V / \mathrm{d} I$ and $\mathrm{d} V / \mathrm{d} H_{\|}$ measured by sweeping the applied $\mathrm{dc}$ magnetic field between 1 and $-5 \mathrm{kOe}$. [(c) and (d)] The $\left(H_{\mathrm{dc}}, I_{\mathrm{dc}}\right)$ phase diagrams for $\mathrm{d} m_{\mathrm{z}} / \mathrm{d} I$ and $\mathrm{d} m_{\mathrm{z}} / \mathrm{d} H_{\|}$from the macrospin simulation in the case of the anisotropy field tilted by $5^{\circ}$. The regions of the $\mathrm{P}$ and $\mathrm{AP}$ states are labeled. change in $H_{\mathrm{dc}}$ or $I_{\mathrm{dc}}$ will then change the angle of the magnetization precession and thus a variation in the resistance. The different signals coming from those measurements can be more clearly shown in the $\left(H_{\mathrm{dc}}, I_{\mathrm{dc}}\right)$ phase diagrams.

Figures $2(\mathrm{a})$ and $2(\mathrm{~b})$ show the $\left(H_{\mathrm{dc}}, I_{\mathrm{dc}}\right)$ phase diagrams for $\mathrm{d} V / \mathrm{d} I$ and $\mathrm{d} V / \mathrm{d} H_{\|}$. The STT-driven precession takes place when $I_{\mathrm{dc}}>5.6 \mathrm{~mA}$ and $H_{\mathrm{dc}}<-1.9 \mathrm{kOe}$. The phase diagrams for $\mathrm{d} V / \mathrm{d} I$ and $\mathrm{d} V / \mathrm{d} H_{\|}$show similar branches which indicate the transition between one steady precession and another. However those transitions are more or less pronounced depending on the kind of excitation. One key difference is the presence of a vertical line around $-1.8 \mathrm{kOe}$ in the $\mathrm{d} V / \mathrm{d} H_{\|}$phase diagram but absent in the $\mathrm{d} V / \mathrm{d} I$ one. It shows that the state that may be probed with $\mathrm{d} V / \mathrm{d} H$ only is affected by the magnetic field and not the current which highlight the fact that these three techniques are complementary. For a better understanding we performed macrospin simulations similar to the one reported in Ref. 10 to compare to the experimental data. Similar parameters were used and the anisotropy axis was by $5^{\circ}$ away from the normal of the film plane. Considering that the voltage changes come from GMR effect only and assuming that the hard layer is solidly fixed along the easy axis then the voltage $V$ is proportional to the magnetization component along the easy axis that will be refereed as $m_{\mathrm{z}}$. As shown in Figs. 2(c) and 2(d), the vertical branch appears in the $\mathrm{d} m_{\mathrm{z}} / \mathrm{d} H_{\|}$phase diagram but cannot be seen in the $\mathrm{d} m_{\mathrm{z}} / \mathrm{d} I$ diagram. The magnetic state related to this effect is a canted state which is a static state for which the magnetization is tilted away from the easy axis.

From this simple macrospin calculation, the plateaus in $R$ versus $H_{\mathrm{dc}}$ and the branches in the precession region of the $\mathrm{d} V / \mathrm{d} I$ phase diagram cannot be explained. These may show the limit of the macrospin model and suggest the presence of nonuniform magnetic configurations.

In summary, we studied STT-driven magnetization dynamics in a nanopillar spin valve with PMA using $R, \mathrm{~d} V / \mathrm{d} I$, $\mathrm{d} V / \mathrm{d} H_{\|}$, and $\mathrm{d} V / \mathrm{d} H_{\perp}$ measurements, respectively. $R$ and $\mathrm{d} V / \mathrm{d} I$ can give the information on hysteretic magnetization switching, and $\mathrm{d} V / \mathrm{d} I, \mathrm{~d} V / \mathrm{d} H_{\|}$, and $\mathrm{d} V / \mathrm{d} H_{\perp}$ can probe re- versible magnetization processes. Both the amplitude and the sign of the peaks in $\mathrm{d} V / \mathrm{d} I, \mathrm{~d} V / \mathrm{d} H_{\|}$, and $\mathrm{d} V / \mathrm{d} H_{\perp}$ show the different influence of current and magnetic field on the magnetization dynamics. The three methods are complementary and offer new ways to study the different magnetic states and their dynamic. More precisely it is shown in this paper that canted states can be easily detected using $\mathrm{d} V / \mathrm{d} H$ whereas it is nearly undetectable using $\mathrm{d} V / \mathrm{d} I$. It should be noted that these low cost and feasible methods are not limited to perpendicular magnetization systems nor to STT devices but it can be used to probe any magnetic state which lead to a MR effect.

This work was partly supported by the ISTRADE project of French National Research Agency (ANR). The authors thank J.Z. Sun and A.D. Kent for fruitful discussions.

${ }^{1}$ J. C. Slonczewski, J. Magn. Magn. Mater. 159, L1 (1996).

${ }^{2}$ L. Berger, Phys. Rev. B 54, 9353 (1996).

${ }^{3}$ M. Tsoi, A. G. M. Jansen, J. Bass, W. C. Chiang, M. Seck, V. Tsoi, and P. Wyder, Phys. Rev. Lett. 80, 4281 (1998).

${ }^{4}$ E. B. Myers, D. C. Ralph, J. A. Katine, R. N. Louie, and R. A. Buhrman, Science 285, 867 (1999).

${ }^{5}$ J. A. Katine, F. J. Albert, R. A. Buhrman, E. B. Myers, and D. C. Ralph, Phys. Rev. Lett. 84, 3149 (2000).

${ }^{6}$ J. Z. Sun, IBM J. Res. Dev. 50, 81 (2006)

${ }^{7}$ S. Mangin, D. Ravelosona, J. A. Katine, M. J. Carey, B. D. Terris, and E. E. Fullerton, Nature Mater. 5, 210 (2006).

${ }^{8}$ D. Ravelosona, S. Mangin, Y. Lemaho, J. A. Katine, B. D. Terris, and E. E. Fullerton, Phys. Rev. Lett. 96, 186604 (2006).

${ }^{9}$ S. Mangin, Y. Henry, D. Ravelosona, J. A. Katine, and E. E. Fullerton, Appl. Phys. Lett. 94, 012502 (2009).

${ }^{10}$ Y. Henry, S. Mangin, J. Cucchiara, J. A. Katine, and E. E. Fullerton, Phys Rev. B 79, 214422 (2009).

${ }^{11}$ J. E. Wegrowe, Q. Anh Nguyen, and T. L. Wade, IEEE Trans. Magn. 46, 866 (2010).

${ }^{12}$ S. Granville, H. Yu, J. Dubois, L. Gravier, and J. P. Ansermet, J. Magn. Magn. Mater. 322, 1464 (2010).

${ }^{13}$ S. Serrano-Guisan, G. D. Domenicantonio, M. Abid, J. P. Abid, M. Hillenkamp, L. Gravier, J. P. Ansermet, and C. Felix, Nature Mater. 5, 730 (2006).

${ }^{14}$ E. E. Fullerton and S. Mangin, Nature Mater. 7, 257 (2008).

${ }^{15}$ B. Dieny, V. S. Speriosu, S. S. P. Parkin, B. A. Gurney, D. R. Wilhoit, and D. Mauri, Phys. Rev. B 43, 1297 (1991). 